\title{
Comparative effects of biomass pre-treatments for direct and indirect transesterification to enhance microalgal lipid recovery
}

\author{
Forough Ghasemi Naghdi ${ }^{1}$, Skye R. Thomas-Hall ${ }^{1}$, Reuben Durairatnam ${ }^{1}$, Steven Pratt $^{2}$ and \\ Peer M. Schenk ${ }^{1 *}$
}

' Algae Biotechnology Laboratory, School of Agriculture and Food Sciences, The University of Queensland, Brisbane, QLD, Australia

${ }^{2}$ School of Chemical Engineering, The University of Queensland, Brisbane, QLD, Australia

\section{Edited by:}

Qiang Wang, Chinese Academy of Sciences, China

Reviewed by:

Petros Katapodis, National University of loannina, Greece

Waqar Majeed, University of Arkansas for Medical Sciences, USA

\section{*Correspondence:}

Peer M. Schenk, Algae Biotechnology Laboratory, School of Agriculture and Food Sciences, The University of Queensland, Level 5, John Hines Building, Brisbane, QLD 4072, Australia

e-mail: p.schenk@uq.edu.au
Microalgal lipid recovery for biodiesel production is currently considered suboptimal, but pre-treatment of algal biomass, the use of solvent mixtures and the positioning of transesterification can lead to increased yields. Here, the effect of various reportedly successful pre-treatments and solvent mixtures were directly compared to each other and combined with direct and indirect transesterification methods using the oleaginous microalga Tetraselmis sp. M8. Microwave and thermal pre-treatments were applied and the total lipid and fatty acid methyl ester (FAME) recoveries were investigated. The application of pretreatments increased FAME recovery through indirect transesterification when a Soxhlet system was used but they had no significant effect for direct transesterification. Gravimetric analyses of total lipids revealed that lipid recovery was highest when utilizing the chloroform-based Bligh and Dyer extraction method; however, FAME yield was the highest when applying a Soxhlet system utilizing a solvent mixture of hexane-ethanol (3:1). Total lipid recovery did not necessarily correlate with the recovery of FAMEs. The highest FAME recovery was achieved from thermal or microwave pre-treated biomass followed by indirect transesterification through Soxhlet extraction. FAME recovery could be more than doubled (increase of up to $171 \%$ ) under these conditions. We conclude that a simple thermal pre-treatment $\left(80^{\circ} \mathrm{C}\right.$ for $\left.10 \mathrm{~min}\right)$ in combination with solvent mixture extraction through indirect transesterification may present a cost-effective and scalable option for large-scale lipid extraction from microalgae.

Keywords: Microalgae, lipid recovery, pre-treatment, direct and indirect transesterification

\section{INTRODUCTION}

Oils from microalgae are an attractive and promising resource for biodiesel production (Amin, 2009; Johnson and Wen, 2009; Demirbas and Fatih Demirbas, 2011) but challenges remain in microalgae harvesting and lipid extraction technologies (Yoo et al., 2012). Fatty acid methyl esters (FAMEs), which are the basis of biodiesel, can be produced by transesterification of algal oil [triacylglycerides (TAGs)] with methanol using either acids or bases as catalysts and resulting in the formation of glycerol as a byproduct (Canakci and Van Gerpen, 1999; Knothe, 2005; Chisti, 2007; Demirbas, 2008). However, the lipids must first be extracted from the microalgal cells, and as the cell walls are generally thick or lipids are associated with organelles or other cellular structures, lipid extraction remains a major bottleneck for algae-derived biodiesel production (Mercer and Armenta, 2011; Yoo et al., 2012). Consequently, there is a demand for environmentally and economically sustainable methods for cell disruption (Zheng et al., 2011) and that are also scalable.

The approaches for cell disruption that have been widely trialed to date range from mechanical methods (such as milling) to chemical (acid/base for cell lysis) and physical (such as sonication, microwave, and thermal) techniques (Kita et al., 2010;
Mercer and Armenta, 2011). These pre-treatments have been shown to break, or at least weaken, the cell wall and internal structures, which consequently facilitated more efficient oil extraction. Lipids are then available for either direct or indirect transesterification. For indirect transesterification, the lipids must first be recovered. Among all of the methods for lipid recovery, the Bligh \& Dyer and the Soxhlet techniques have been used widely as benchmarks (Bligh and Dyer, 1959, Luque-Garci'a and Luque de Castro, 2004; Mercer and Armenta, 2011). But direct transesterification, which has been considered as a candidate for large-scale biodiesel production, has been reported to be at least $15-20 \%$ more efficient over extraction-transesterification experiments (indirect transesterification) (Lewis et al., 2000; Levine et al., 2010).

Microwave was previously identified as the most promising pre-treatment (Virot et al., 2007; Cravotto et al., 2008; Balasubramanian et al., 2011). Most of the pre-treatments previously carried out are not suitable for large-scale applications or are too expensive. The objective of this study was to evaluate the efficiency of indirect transesterification (through applying Bligh \& Dyer and Soxhlet extraction techniques) along with direct transesterification, both coupled with thermal and microwave pre-treatments on lipid extraction from a high-lipid producing marine green alga 
(Tetraselmis sp. M8). This study was designed to fill the gap of such a comparison between different lipid extraction/recovery techniques coupled with different types of pre-treatments in related research fields. Not only the amount of extracted lipids was considered, but also the potential effect of the extraction-recovery procedures on the lipid class and fatty acid composition were analyzed. The results of this study may assist in optimizing the production of biodiesel from microalgae.

\section{MATERIALS AND METHODS MICROALGAE CULTIVATION}

Tetraselmis sp. strain M8 (Chlamydomonadaceae), was isolated from a rock pool at the Sunshine Coast $\left(26^{\circ} 39^{\prime} 39^{\prime \prime} \mathrm{S}, 153^{\circ} 6^{\prime} 18^{\prime \prime} \mathrm{E}\right)$ and was cultured in the Algae Biotechnology Laboratory at the University of Queensland (Lim et al., 2012). It was grown in f/2 medium (Guillard and Ryther, 1962) in artificial sea water and then scaled up to a $1000 \mathrm{~L}$ outdoor open raceway pond equipped with an airlift mechanism for mixing. Microalgae were harvested by centrifugation after 5 days of nitrogen starvation to provide a highlipid content biomass. The biomass for the initial baseline experiments was provided by a $10 \mathrm{~L}$ laboratory-grown culture (using clear 20 polyethylene bags and air bubbling under 16:8 h day: night illumination); while for the direct and indirect transesterification experiments, the outdoor raceway culture was used.

\section{SYTOX GREEN STAINING}

Five millimolar stock solution of SYTOX Green in DMSO (Invitrogen, Ltd., UK) was used for staining of damaged cells. A total of $0.5 \mu \mathrm{L}$ of this solution was used for staining $0.5 \mathrm{~mL}$ of cell suspensions followed by $5 \mathrm{~min}$ incubation at room temperature in the dark. The observation of the stained cells was carried out through utilizing a fluorescence microscope (BX61, Olympus, Japan).

\section{NILE RED STAINING}

Nile red (Sigma-Aldrich) was prepared by dissolving $10 \mathrm{mg}$ of Nile red powder in $1 \mathrm{~mL}$ acetone and stored in the dark at $4^{\circ} \mathrm{C}$ as a $31.41 \mathrm{mM}$ stock solution. TAG observation was conducted using an Olympus BX60 fluorescent microscope with an Olympus U-RFL-T burner.

\section{MICROALGAE HARVESTING AND PROCESSING}

An Avanti centrifuge (HP-20 XPI) (Beckman Coulter) was used for centrifugation of volumes higher than $1 \mathrm{~L}$ and a Techno Spin $\mathrm{R}$ centrifuge (Sorvall Instruments) was used for small amounts $(<100 \mathrm{~mL})$. Lyophilization was carried out by using a DYNAVAC Freeze drying unit model FDI. For turning the dried biomass to fine powder, milling was accomplished by a Retsch NM 301 mixer mill machine.

The biomass preparation was decided as per the extraction experiments on both lyophilized dry biomass and wet concentrated algal paste. The best solvent composition for Soxhlet extraction was selected based on the results of the Soxhlet extraction through utilizing hexane or a solvent mixture of hexane-ethanol (3:1 ratio). The pre-treatments were carried on Tetraselmis sp. M8 followed by direct and indirect transesterification as summarized below. The procedures of the experiments are described in Figure 1.

\section{PRE-TREATMENTS OF ALGAL BIOMASS}

Tetraselmis sp. M8 slurry from the same batch was used for comparisons within an experiment.

\section{Thermal}

Two liters of Tetraselmis sp. M8 slurry (0.8 gram dry weight (DW)/L) were transferred to an Erlenmeyer flask and subsequently placed on a hot plate with a magnetic stirrer. The culture was heated up to $80^{\circ} \mathrm{C}$ and kept at this temperature for $10 \mathrm{~min}$, while it was continuously stirred.

\section{Microwave}

A 1 L-beaker was filled with Tetraselmis sp. M8 slurry (0.8 g DW/L) and placed in a microwave (LG Microwave Oven Model no. MS3447GR) at a setting of $1.1 \mathrm{~kW}$ for $3 \mathrm{~min}$. The slurry was then stirred to a homogenous mixture and immediately placed in the microwave under the same settings for another $2 \mathrm{~min}$. The temperature of the slurry after the microwave treatment ranged between 80 and $90^{\circ} \mathrm{C}$.

The slurries from above pre-treatments as well as the untreated control were centrifuged at $4000 \times g$ for $7 \mathrm{~min}$ and then lyophilized followed by milling for $20 \mathrm{~s}$ at a frequency of $20(1 / \mathrm{s})$.

\section{LIPID EXTRACTION}

Lipid extraction from microalgae was carried out following either the procedure by Bligh and Dyer (1959) or by using a Soxhlet (Foss Soxtec 1043). For lipid extraction according to Bligh \& Dyer, $1 \mathrm{~g}$ of lyophilized Tetraselmis sp. M8 or the equivalent DW of paste were placed in $40 \mathrm{~mL}$ glass vials with Polytetrafluoroethylene (PTFE) lined caps. Five milliliters chloroform and $10 \mathrm{~mL}$ methanol were then added and the mixture was vortexed for $5 \mathrm{~min}$. Then an additional $5 \mathrm{~mL}$ chloroform was added and vortexing was conducted for another $5 \mathrm{~min}$ followed by addition of distilled water (the amount of distilled water addition was based as per the moisture content of the biomass to result in a ratio of 2:2:1.8 of chloroform/methanol/distilled water as detailed by Bligh and Dyer). Then centrifugation at $1000 \times g$ for $7 \mathrm{~min}$ was conducted to form phase separation, and subsequently the organic layer (chloroform with extracted lipids) was transferred to pre-weighted glass tubes and the chloroform was evaporated using a vacuum desiccator.

Soxhlet extraction with hexane or hexane-ethanol was carried out using a Foss Soxtec 1043 equipped with six extraction chambers and valves for gathering and recycling the distilled solvent. Solvents used were hexane, methanol, chloroform, and ethanol; all were HPLC grade sourced from Merck KGaA. Each of the six cellulose thimbles was loaded with about $0.7 \mathrm{~g}$ of lyophilized Tetraselmis sp. M8 biomass, which were placed in pre-weighted aluminum cups previously filled with either $52 \mathrm{~mL}$ hexane or a hexane-ethanol $(3: 1 ; \mathrm{v} / \mathrm{v})$ solvent mixture. The extraction (solvent circulation) was performed for $6 \mathrm{~h}$ followed by $30 \mathrm{~min}$ rinsing and 30 min evaporation. Weighing the samples and gravimetric measuring of the lipid extractions were carried out using a digital Mettler AM50 scale with $1 \mathrm{mg}$ accuracy.

\section{FATTY ACID PROFILING AND QUANTIFICATION}

For direct transesterification, $4 \mathrm{mg}$ of lyophilized algal pellet was hydrolyzed and methyl-esterified with $300 \mu \mathrm{L}$ of $2 \% \mathrm{H}_{2} \mathrm{SO}_{4}$ in 


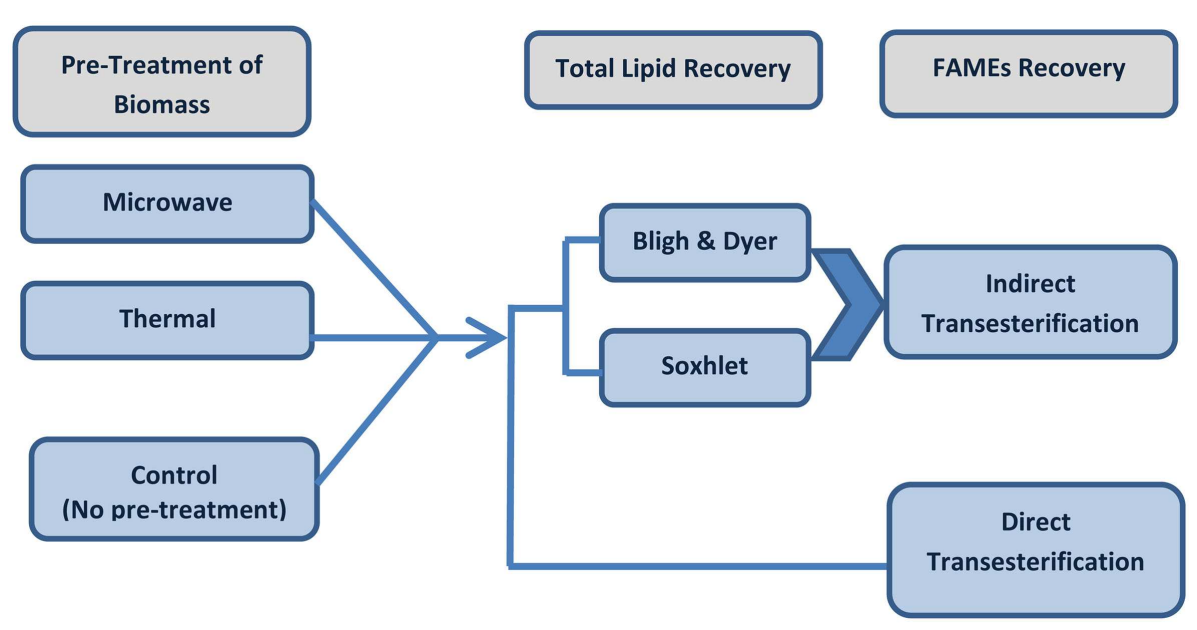

FIGURE 1 | Schematic experimental flow of pre-treated or untreated (control) algal biomass for lipid extraction followed by direct or indirect transesterification techniques

methanol solution at $80^{\circ} \mathrm{C}$ by shaking $(480 \mathrm{rpm})$ for $2 \mathrm{~h}$ on a thermal-mixer. Prior to the esterification, $50 \mu \mathrm{g}$ of heneicosanoic acid (C21) was added to the pellet in each sample as an internal standard. After esterification, $300 \mu \mathrm{L}$ of $0.9 \%(\mathrm{w} / \mathrm{v}) \mathrm{NaCl}$ and $300 \mu \mathrm{L}$ of HPLC grade hexane were added and vortexed for $20 \mathrm{~s}$. Phase separation was performed by centrifugation at $16,000 \times g$ for $3 \mathrm{~min}$ and the hexane layer was used for FAME profile analysis by GC-MS.

Indirect transesterification was conducted by applying two extraction techniques (Bligh \& Dyer or Soxhlet) followed by transesterification of the recovered triglycerides for GC-MS. Bligh \& Dyer extraction was performed on $1 \mathrm{~g}$ lyophilized Tetraselmis $\mathrm{sp}$. M8 biomass. The Soxhlet extraction technique was conducted by utilizing a solvent mixture of hexane-ethanol with a ratio of 3:1 on $0.7 \mathrm{~g}$ lyophilized Tetraselmis sp. M8. Both extraction techniques were carried out as mentioned for the baseline experiments.

The extracted lipids were re-dissolved in $2 \mathrm{~mL}$ chloroform and a $100 \mu \mathrm{L}$ aliquot was taken and dried down. Then the acid catalyzed derivatization was performed (similarly to what has been described in detail for the direct transesterification). Using $1 \mu \mathrm{L}$ on a splitless injection system, GC-MS analyses were carried out on an Agilent 6890 GC coupled to a 5975 MSD. A DB-Wax column (Agilent, 122-7032) was used with running conditions as described in Agilent's RTL DB-Wax method (Application note: 5988-5871EN). Identification of FAMEs was based on mass spectral profiles and retention times in the Agilent's RTL DB-Wax method.

\section{TEST FOR LIPIDS POTENTIALLY RELEASED TO THE MEDIUM AFTER PRE-TREATMENTS OF ALGAL BIOMASS}

To quantify lipids potentially released to the medium after pretreatments of algal biomass, $500 \mathrm{~mL}$ of slurry from each of the pre-treatments (including the untreated control), were placed in $500 \mathrm{~mL}$ Borosilicate glass volumetric flasks and were kept at $4^{\circ} \mathrm{C}$ overnight so that algal biomass completely settled and cooled down, allowing the accumulation of any released floating lipids on top of the supernatant. Then from each flask, $50 \mathrm{~mL}$ of the very top layer of the supernatant was transferred as $10 \mathrm{~mL}$ portions to glass vials for subsequent Bligh \& Dyer extraction. Then, the settled slurries were shaken again to form homogenous slurries and from each, $50 \mathrm{~mL}$ were centrifuged at a high speed of $4000 \times g$ for $15 \mathrm{~min}$ and the supernatant was transferred in $10 \mathrm{~mL}$ portions to glass vials for subsequent Bligh \& Dyer extraction. A modified Bligh \& Dyer extraction was conducted by adding $5 \mathrm{~mL}$ chloroform followed by $5 \mathrm{~min}$ vortexing, then $11 \mathrm{~mL}$ methanol were added followed by $1 \mathrm{~min}$ vortexing and lastly $6 \mathrm{~mL}$ chloroform were added and a final step of 5 min vortexing was carried out. The rate of solvents was selected in a way to form the ratio of 2:2:1.8 of chloroform: methanol: water in the end as has been suggested by Bligh and Dyer (1959). Then from the readily separated phases, the bottom layer of chloroform with any dissolved lipids was transferred to pre-weighted soda glasses for gravimetric analyses. All extraction experiments were carried out in triplicates.

\section{STATISTICAL ANALYSIS}

The extracted lipid and FAMEs were compared according to different samples (algal paste and lyophilized biomass), different solvent systems, various pre-treatment systems, or different extraction methods using $t$ test, one-way, and two-way ANOVA methods. The level of significant difference was at $P<0.05$. Statistical analyses were conducted through GraphPad Prism 6.

\section{RESULTS}

DRIED ALGAL BIOMASS YIELDS MORE LIPIDS THAN WET BIOMASS

Lipid extraction is currently considered a bottleneck in commercial algae cultivation for biodiesel production. One of the first questions is whether drying of algal biomass is required for efficient lipid extraction or whether extraction from wet biomass is possible. A comparison of the lipid extraction efficiency using the Bligh \& Dyer method showed that algal paste (with a moisture content of $80.8 \%$ ) was still suitable for lipid extraction, but yielded $32 \%$ less lipids than lyophilized dry biomass of Tetraselmis sp. M8 (Figure 2). The total lipid recovery from lyophilized biomass was $12.35 \pm 0.24 \%$ of the DW, whereas, the same extraction method on paste of the same biomass showed a lower extraction efficiency 

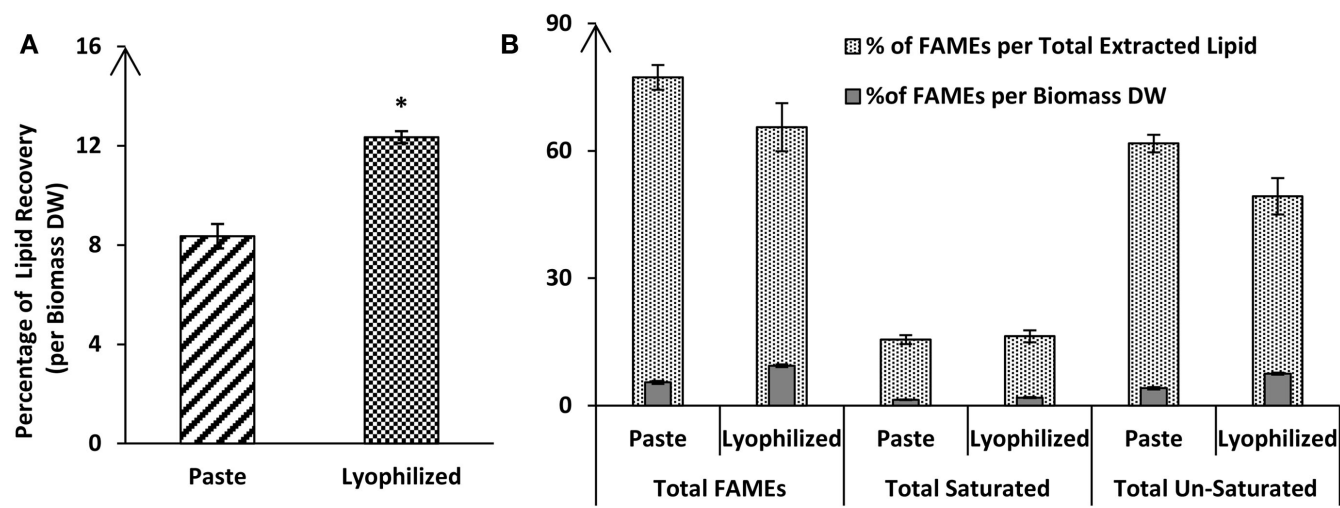

FIGURE 2 | Percentage of total lipid recovery (A) or fatty acid recovery (B) from lyophilized or wet (paste) algal biomass of Tetraselmis sp. M8 using the Bligh \& Dyer extraction method. All values are shown mean lipid contents $( \pm S D)$ relative to biomass dry weight $(D W)$ and/or total extracted lipids from three independent extractions. Asterisks represent significant statistical differences $(P<0.05)$ between lyophilized a wet algal biomass.

Table 1 | Quantitative fatty acid profiles of extracted lipids from wet algal paste or lyophilized biomass of Tetraselmis sp. M8 using the Bligh \& Dyer extraction method followed by FAME analysis using GC-MS.

\begin{tabular}{lccccc}
\hline & \multicolumn{1}{c}{ Lyophilized } & Paste & & Lyophilized & Paste \\
\hline C15 & ND & $1.02 \pm 0(1.9 \%)$ & C18:3 & $16.51 \pm 1.13(17.6 \%)$ & $9.58 \pm 0.83(17.5 \%)$ \\
C16 & $18.05 \pm 0.22(19.2 \%)$ & $12.37 \pm 0.75(22.6 \%)$ & C18:4 & $2.8 \pm 0.06(3 \%)$ & $1.47 \pm 0.16(2.7 \%)$ \\
C16:1 & $2.98 \pm 0.05(3.2 \%)$ & $1.83 \pm 0.06(3.3 \%)$ & C20:4 & $2.72 \pm 0.04(2.9 \%)$ & $1.91 \pm 0.64(3.5 \%)$ \\
C16:3 & $6.93 \pm 0.12(7.4 \%)$ & $3.37 \pm 0.27(6.2 \%)$ & C20:5 & $4.28 \pm 0.11(4.6 \%)$ & $2.25 \pm 0.43(4.1 \%)$ \\
C16:4 & $14.78 \pm 0.5(15.7 \%)$ & $6.5 \pm 0.68(11.9 \%)$ & Others & $0.84 \pm 1.05(0.9 \%)$ & $0.06 \pm 0.09(0.1 \%)$ \\
C18 & $0.29 \pm 0.08(0.3 \%)$ & $0.23 \pm 0.12(0.4 \%)$ & Total FAMEs & $94.04 \pm 3.37(100 \%)$ & $54.79 \pm 3.58(100 \%)$ \\
C18:1 & $7.17 \pm 0.02(7.6 \%)$ & $4.69 \pm 0.17(8.6 \%)$ & Total saturated & $18.94 \pm 1.23(20.1 \%)$ & $13.63 \pm 0.87(24.9 \%)$ \\
C18:2 & $16.69 \pm 0.41(17.7 \%)$ & $9.49 \pm 0.64(17.3 \%)$ & Total un-saturated & $75.1 \pm 2.31(79.9 \%)$ & $41.16 \pm 2.71(75.1 \%)$ \\
\hline
\end{tabular}

Values are means (milligram of FAMEs per gram of biomass $D W) \pm S D$ from three independent extractions.

(): Fatty acid composition (\% $w / W$ ).

$(8.36 \pm 0.49 \%$ of DW; $P<0.05)$. Fatty acid profiling by GC-MS showed that for almost all of the individual fatty acids, there was a considerable difference between the paste and lyophilized biomass.

Interestingly, although the amounts of fatty acids were different (lyophilized biomass showed considerable higher values compared to algal paste), they both resulted in similar fatty acid profiles. Nonetheless, the FAME recovery from the total extracted lipid of the lyophilized algal biomass was $77.31 \pm 2.94 \%$, while the FAME recovery from total extracted lipid from algal paste was only $65.59 \pm 5.69 \%(P>0.05)$ (Figure 2$)$. The percentage of total saturated fatty acids from the total extracted lipid of the two samples were almost the same (around 16\%), but lyophilized biomass showed higher amounts of total un-saturated fatty acids per biomass DW in comparison with algal paste $(7.51 \pm 0.231 \%$, $4.116 \pm 0.271$, respectively; $P<0.05$ ) (Figure 2). Individual FAME profiles are shown in Table 1.

\section{USE OF A SOLVENT MIXTURE CAN INCREASE YIELDS OF TOTAL FAMES}

Hexane is a common solvent for larger scale lipid extraction from biomass that is often used for vegetable oil extraction. However, Chen et al. (2012) tested different ratios of hexane to ethanol and reported that a mixture of hexane:ethanol with the ratio of $(3: 1)$ gave the best results. To test whether a solvent mixture may also lead to increased lipid extraction efficiencies for Tetraselmis sp. M8, a Soxhlet extraction was used. Lipid extraction using hexane led to $7.74 \pm 0.68 \%$ extraction of total lipids from lyophilized algal biomass while there was a much higher lipid recovery for the Soxhlet utilizing a mixture of hexane-ethanol with ratio of $3: 1(14.31 \pm 0.25 \% ; P<0.05$; Figure 3). Interestingly, FAME profiling by GC-MS showed that not only the total lipid recovery increased by applying the hexane-ethanol (3:1) solvent mixture, but it also contained a higher percentage of fatty acids. The total fatty acids extraction rate by the solvent mixture as per the biomass dried weight was significantly higher than when the plain hexane extraction was used $(7.372 \pm 0.372 \%$ and $3.949 \pm 0.238 \%$, respectively; $P<0.05$ ) (Figure 3). On the other hand, the C16 content comprised about $21 \%$ of the total fatty acids when using the solvent mixture, whereas, when plain hexane was used total fatty acids just included $14.6 \%$ of C16 (Table 2). Interestingly, from the total extracted lipid, the percentages of the total fatty acids were near identical for the two solvent categories $(51.54 \pm 3.25$ and $51.12 \pm 1.71 \%$ for hexane-ethanol (3:1) and plain hexane, respectively) (Figure 3). The proportion of total un-saturated FAMEs compared to total FAMEs was higher in the plain hexane Soxhlet 


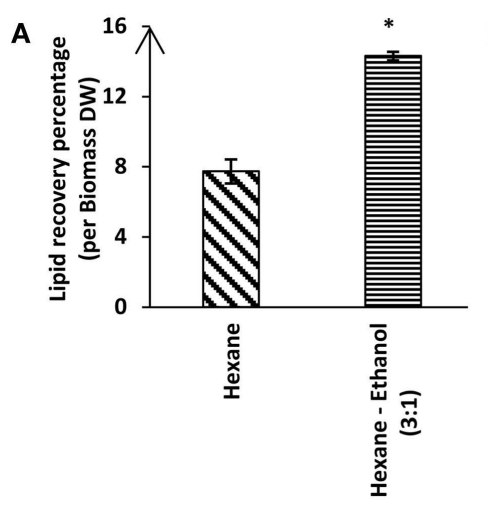

FIGURE 3 | Percentage of total lipid recovery (A) or fatty acid recovery (B) from lyophilized Tetraselmis sp. M8 biomass using hexane or hexane-ethanol (3:1) Soxhlet extraction. All values are

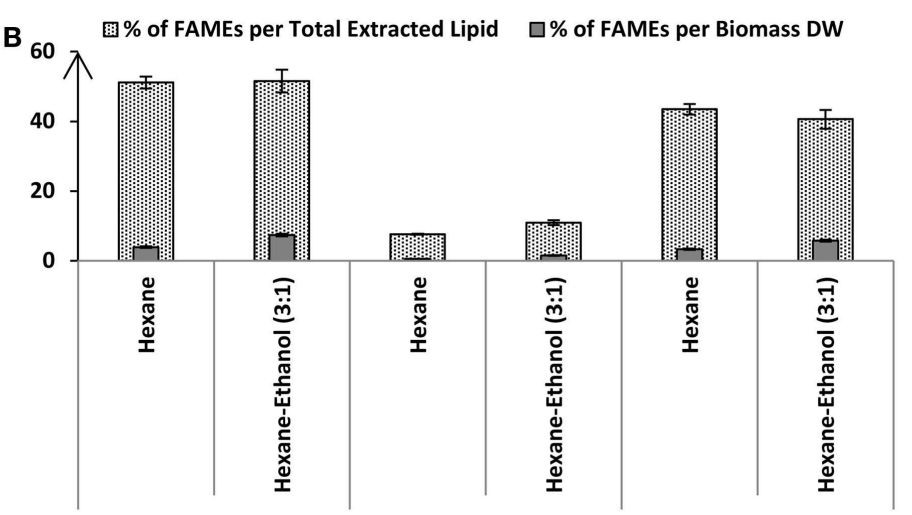

Table 2 | Quantitative fatty acid profiles determined from FAME analyses by GC-MS of Soxhlet-extracted lipids from lyophilized biomass of Tetraselmis sp. M8 using hexane or hexane-ethanol (3:1).

\begin{tabular}{|c|c|c|c|c|c|}
\hline & Hexane & Hexane-ethanol (3:1) & & Hexane & Hexane-ethanol (3:1) \\
\hline C16 & $5.75 \pm 0.42(14.6 \%)$ & $15.35 \pm 0.92(20.8 \%)$ & C18:3 & $7.28 \pm 0.34(18.4 \%)$ & $13.47 \pm 0.61(18.3 \%)$ \\
\hline C16:1 & $0.93 \pm 0.09(2.4 \%)$ & $2.2 \pm 0.15(3 \%)$ & C18:4 & $1.3 \pm 0.13(3.3 \%)$ & $2.21 \pm 0.16(3 \%)$ \\
\hline C16:3 & $3.22 \pm 0.18(8.2 \%)$ & $4.97 \pm 0.35(6.7 \%)$ & C20:4 & $1.54 \pm 0.07(3.9 \%)$ & $2.22 \pm 0.08(3 \%)$ \\
\hline C16:4 & $7.07 \pm 0.52(17.9 \%)$ & $10.36 \pm 1.03(14.1 \%)$ & C20:5 & $2.5 \pm 0.12(6.3 \%)$ & $3.71 \pm 0.17(5 \%)$ \\
\hline C18 & $0.16 \pm 0.01(0.4 \%)$ & $0.32 \pm 0.04(0.4 \%)$ & Total FAMEs & $39.49 \pm 2.38(100 \%)$ & $73.72 \pm 3.72(100 \%)$ \\
\hline C18:1 & $2.88 \pm 0.18(7.3 \%)$ & $5.66 \pm 0.29(7.7 \%)$ & Total saturated & $5.91 \pm 0.43(15 \%)$ & $15.67 \pm 0.9(21.3 \%)$ \\
\hline C18:2 & $6.86 \pm 0.36(17.4 \%)$ & $13.24 \pm 0.64(18 \%)$ & Total un-saturated & $33.58 \pm 1.95(85 \%)$ & $58.05 \pm 3.07(78.7 \%)$ \\
\hline
\end{tabular}

Values are means (milligram of FAMEs per gram biomass DW) $\pm S D$ from three independent extractions.

(): Fatty acid composition (\% w/W).

extraction compared to when the solvent mixture was used (85 and $78.7 \%$, respectively; $P<0.05$ ) (Figure 3). Individual FAME profiles are shown in Table 2.

\section{PRE-TREATMENTS OF ALGAL BIOMASS SIGNIFICANTLY INCREASED LIPID AND FAME RECOVERY}

Cell walls and cellular structures can potentially hinder solvent penetration leading to suboptimal lipid extraction efficiencies. To test this, we evaluated the effect of different cell-disrupting pre-treatments on the efficiency of total lipids/FAMEs recovery of lyophilized algal biomass through either direct or indirect transesterification techniques. Sixty liters of nitrogen-starved Tetraselmis sp. M8 slurry were harvested from an open raceway pond at the same time to provide enough biomass grown under the same conditions for all subsequent experiments to make a direct comparison of the results possible. As has been shown by baseline experiments, lyophilized Tetraselmis sp. M8 biomass showed better oil yield compared to wet algal paste (Figure 2). Hence, for all subsequent experiments, lyophilized biomass was used. Moreover, as the Soxhlet extraction with a solvent mixture using hexane-ethanol (3:1) showed more promising results than the Soxhlet extraction using plain hexane (Figure 3), the solvent mixture was used. Figure 1 shows an overview of the process of these experiments. shown relative to dry weight from three independent extractions. Asterisks represent significant statistical differences of the different solvents used $(P<0.05)$.
Pre-treatments of algal biomass were chosen based on their potential scalability and ability to disrupt cells. Microscopic analyses showed that thermal and microwave pre-treatments led to effective cell disruption and were hence further investigated for their potential to improve lipid extraction efficiency. Figure 4 shows the total lipid recovery of lyophilized Tetraselmis sp. M8 biomass by applying indirect transesterification through Soxhlet or Bligh \& Dyer extraction methods using different pre-treatments. The Bligh \& Dyer method showed better oil recoveries than the Soxhlet method in all of the experiments. Considering just Bligh \& Dyer extractions, the total lipid recovery percentage of the untreated M8 control samples was $18.46 \pm 0.43 \%$, but significantly higher $(P<0.05)$ lipid yields were gained from the microwave or thermal pre-treated biomass $(23.36 \pm 0.23$ and $22.29 \pm 0.23 \%$, respectively).

Going through the Soxhlet results, the same order of extraction efficiency can be found, with the microwave and thermal pre-treatments giving the best oil yields of $20.26 \pm 0.08$ and $20.04 \pm 0.41 \%$, respectively, while the untreated control sample showed only minimal amount of $14.38 \pm 0.93 \%$ lipid recovery.

Fatty acid profiling by GC-MS was performed for both extraction methods by indirect transesterification or direct transesterification for each of the pre-treatment systems; the obtained data are 


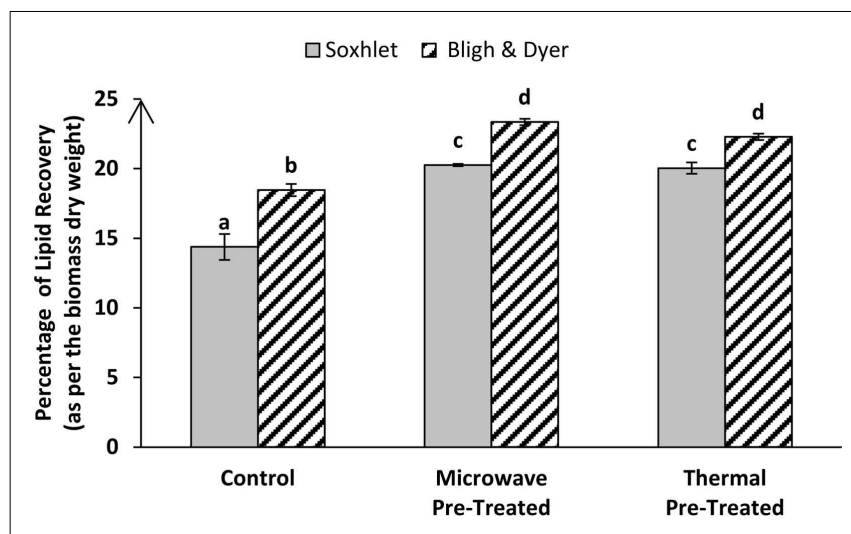

FIGURE 4 | Percentage of total lipid recovery per dry weight from Tetraselmis sp. M8 algal biomass through Bligh \& Dyer and Soxhlet extraction of pre-treated or untreated (control) samples. Different letters represent significant statistical differences $(P<0.05)$.

shown in Table 3. All of the methods resulted in similar fatty acid profiles, with $\mathrm{C} 16, \mathrm{C} 16: 4$, and $\mathrm{C} 18: 3$ contributing to more than half of the FAMEs in total.

Comparisons of fatty acids recovery for the different pretreatment systems and extraction methods are shown in Figure 5. The untreated control samples of both direct and indirect transesterification experiments showed similar total FAME contents per DW. It is interesting to note that although Soxhlet extraction showed lower amounts of total lipid recovery than the Bligh \& Dyer-based extraction, it was significantly more efficient for the total yield of FAMEs for samples that underwent thermal pretreatment $(P<0.05)$. For both indirect transesterification procedures (following lipid extraction by Bligh \& Dyer or Soxhlet), thermal and microwave pre-treatment gave significantly higher FAME yields. The highest yield of un-saturated fatty acids per DW was obtained when using the Soxhlet extraction after thermal or microwave pre-treatment (Table 3).

Within the indirect transesterification experiments, considering the total FAME recovery from the total extracted lipid, the best result was obtained by Soxhlet extraction of both thermal and microwave-pre-treated biomass with $86.06 \pm 6.29$ and $74.25 \pm 11.1 \%$ recovery rates, respectively.

\section{INTRACELLULAR LIPIDS ARE NOT MARKEDLY RELEASED DUE TO PRE-TREATMENTS}

The main reason for applying the pre-treatments was to facilitate the extraction techniques by weakening microalgal cell walls so that the solvents can more readily permeate the cells to subsequently dissolve and extract the lipids. Hence, there was a concern that whether due to the pre-treatments, microalgal cells had collapsed and some of the intracellular lipids were released to the medium (seawater) and therefore were removed from the extraction system during centrifugation, which could result in under-estimation of the lipid recovery. However, microscopic analyses, including SYTOX Green staining of pre-treated algal slurries showed intact cells with no visible lipid bodies in the medium (Figure 6).
Microscopic visualizing the Nile Red stained samples of the supernatants of the pre-treatment systems under the UV light showed nothing more than a plain black background. To test larger volumes of the medium surrounding pre-treated algal cells, $50 \mathrm{~mL}$ of the supernatant from settled algal biomass was extracted via Bligh \& Dyer. The results confirmed that there was no detectable lipid recovery from the supernatants of the pre-treatment systems. Hence, the aforementioned results can be considered as the precise total lipid extractions without significant losses.

\section{DISCUSSION}

This study emphasizes the importance of pre-treatments, use of solvents and lipid extraction techniques for microalgal oil extraction. Significant yield improvements can be achieved by variations and combinations of these simple parameters. For example, drying of algal biomass by lyophilization resulted in higher total lipid yields, but interestingly also a higher recovery of fatty acids derived from TAGs (Figure 2). This latter result is particularly relevant for the potential production of microalgal biodiesel, as only this portion of the lipids is considered suitable for transesterification (Levine et al., 2010). Fatty acid composition was mostly unaffected, as FAME profiles obtained by GC-MS after Bligh \& Dyer extraction on both wet and dried biomass resulted in similar fatty acid profiles (Table 1). The presence of moisture in the algal biomass has been reported to be limiting the lipid recovery and was suggested to be caused by the barrier action of the water between the algal cells and hydrophobic solvent (Lee et al., 2010; Balasubramanian et al., 2011; Patil et al., 2011; Wahlen et al., 2011). Hence, although the drying process of harvested microalgae is known to consume significant amounts of energy and even just $25 \%$ reduction in the drying process can increase the energy balance considerably (Lardon et al., 2009; Xu et al., 2011), the higher recovery of the lipids from the lyophilized microalgae might compensate for the additional costs. Alternatively, solar air drying might be used to reduce energy input. Another advantage of using dry algae is the option to store and transport algal biomass. In addition, dried samples can be handled and weighted much more easily and lipases, which can cause lipolysis and are potentially present, would be deactivated by water removal (Ryckebosch et al., 2012). Drying can also be regarded as a pre-treatment that causes cell rupture and this may contribute to the improved lipid extraction efficiency compared to when wet biomass is used. Future studies may focus on optimizing the best drying conditions while using minimal energy input.

Soxhlet-facilitated lipid extraction is one of the most commonly used methods for microalgal lipid extraction and has been referred to as a benchmark for comparisons to other extraction techniques (Luque-Garci'a and Luque de Castro, 2004). The target compound polarity influences the choice of solvents for lipid extraction (Pérez-Serradilla et al., 2007). Hexane has been extensively used for lipid extraction by the Soxhlet method. This low-cost hazardous solvent is known to have a high solubility for non-polar TAGs, however, its efficiency in lipid recovery can be increased significantly by mixing it with a polar solvent (Gandhi et al., 2003; Virot et al., 2007; Balasubramanian et al., 2011; Yoo et al., 2012). This was also confirmed by the present study where the use of a hexane-ethanol (3:1) mixture, rather than plain hexane, significantly improved lipid extraction efficiency (Figure 3A). Ethanol 


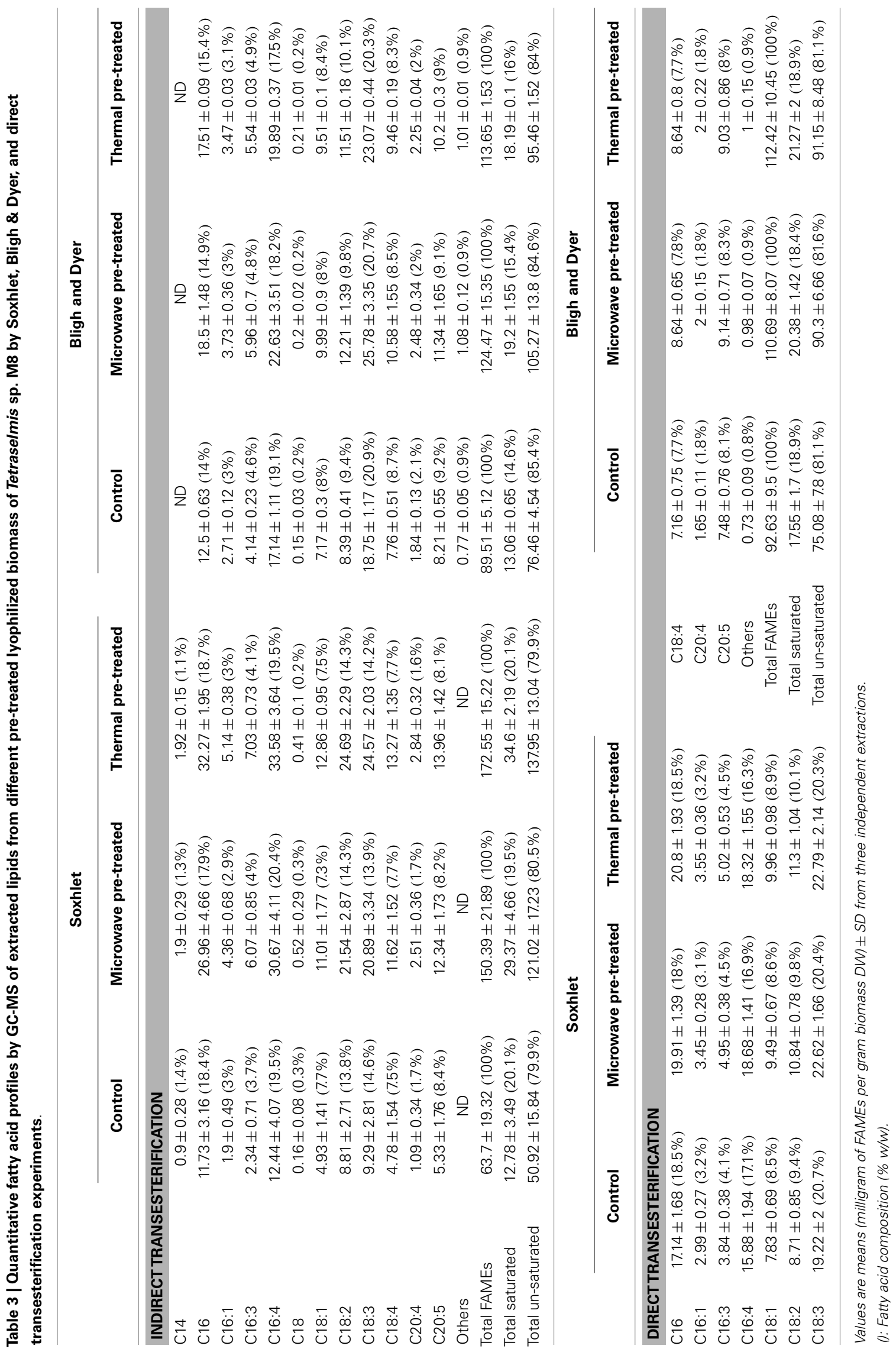


has been selected due to its similar evaporation rate with hexane. Moreover, in the sub-critical co-solvent lipid extraction from wet Nannochloropsis sp., the ratio of 3:1 of hexane to ethanol proved to be an appropriate solvent mixture (Chen et al., 2012). Ethanol was reported to result in significantly higher yield than hexane in extracting the lipids from Scenedesmus obliquus $(62.04 \pm 2.42$ and $40.71 \pm 4.46 \%$ per dry mass, respectively) (Balasubramanian et al., 2011). One explanation for the increased lipid recovery from the solvent mixture could be that ethanol would extract polar lipids and other lipophilic molecules that would otherwise not be extractable by hexane only. However, these additional compounds would not contribute to feedstock quality for biodiesel production

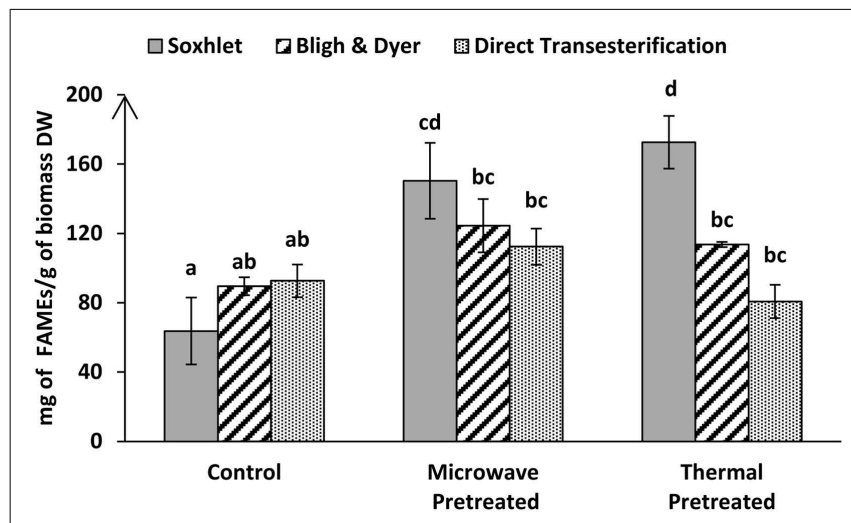

FIGURE 5 | Total FAME recoveries obtained by GC-MS from lyophilized Tetraselmis sp. M8 biomass via indirect transesterification through Bligh \& Dyer or Soxhlet and direct transesterification using different pre-treatment systems. Different letters represent significant statistical differences $(P<0.05)$. and indeed could inhibit transesterification (Moser, 2011; Sathish and Sims, 2012). However, our results show that, apart from the increased total lipid recovery, the rate of total FAMEs has also been increased proportionally by over $50 \%$ when using the solvent mixture for extraction (Figure 3B; Table 2). It has been suggested that a solvent mixture containing a polar solvent may be more capable of releasing lipids from their protein-lipid complexes followed by subsequent dissolving of the lipids in the non-polar solvent (Ryckebosch et al., 2012). On the other hand, ethanol can be easily saturated due to its lower solubility to lipids but its extraction efficiency can be promoted when added to hexane. In this view, the ethanol molecules carry the lipids between algal cells and hexane, as they can diffuse through the microalgal cells and transfer the extracted lipids to hexane (Chen et al., 2012). Alternatively, ethanol may also generally help with the disruption of cellular structures and membranes, which would allow better penetration of hexane to reach more cellular lipids.

Microalgal lipid extraction is hindered by their rigid cell walls (Hejazi and Wijffels, 2004) and hence, an appropriate cell disruption method and device has been suggested to be key to increase the lipid extraction efficiency (Lee et al., 2010). The pretreatments used in the present study have improved the efficiency of both, total lipid and total FAME recovery for indirect transesterification techniques (Figures 4 and 5). This result led to the hypothesis that although Bligh \& Dyer is thought to extract total lipids, it may provide an underestimate if applied on unpre-treated algal biomass. The main reason for this could be that although Bligh \& Dyer extraction is known for successful extraction of almost $100 \%$ of total lipid from fish tissue, the lipid recovery from microalgae could be much lower, mainly due to the unusual lipid classes and fatty acids found within microalgal cells, which are different from those in animal organisms

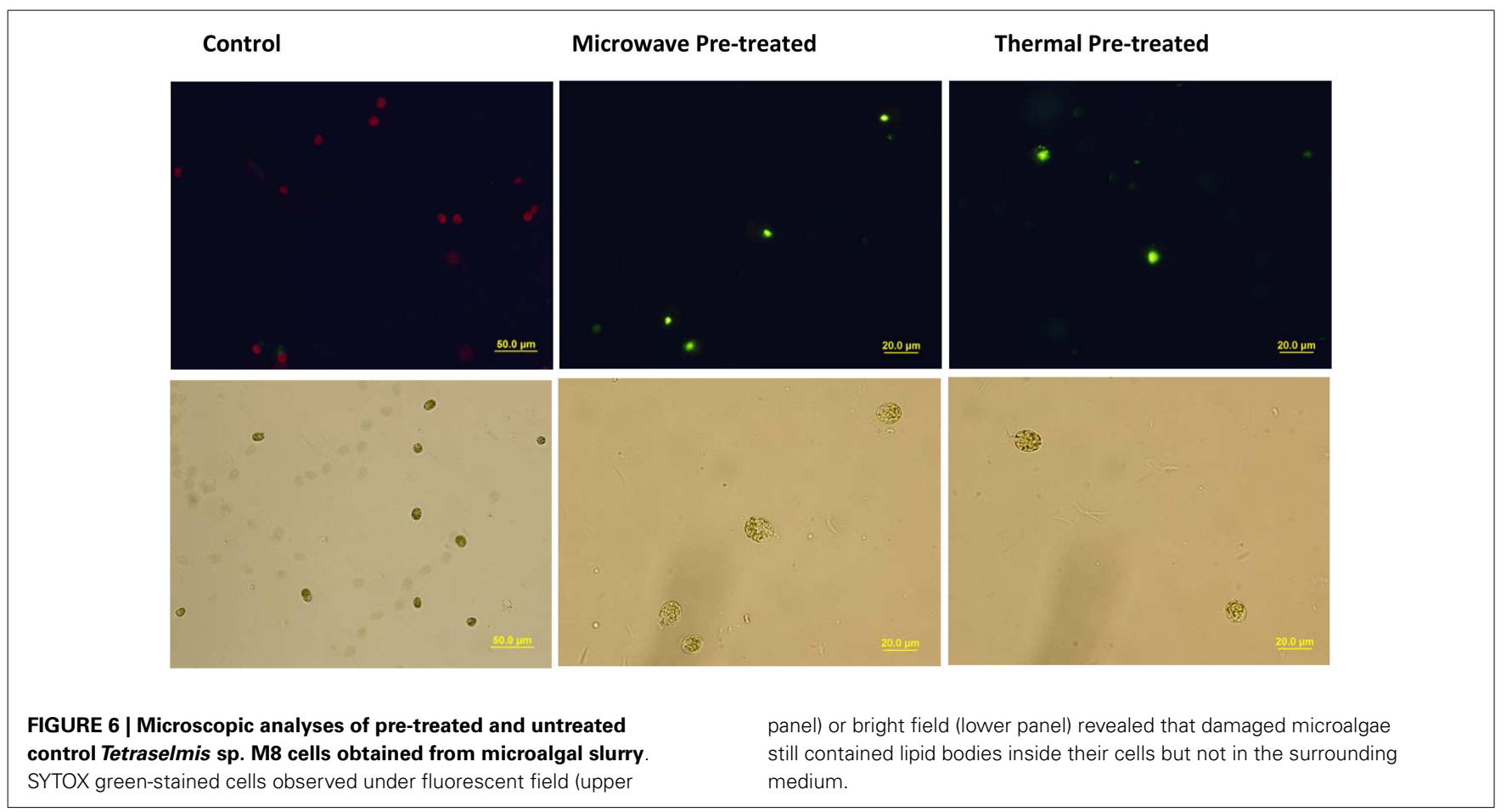


Table 4 | Percentage of total FAME recovery from total extracted lipid

\begin{tabular}{llll}
\hline & \multicolumn{1}{c}{ Control } & Microwave & Thermal \\
\hline Soxhlet & $44.57 \pm 14.77^{\mathrm{a}}$ & $74.25 \pm 11.1^{\mathrm{b}, \mathrm{c}}$ & $86.06 \pm 6.29^{\mathrm{c}}$ \\
Bligh \& Dyer & $48.34 \pm 2.81^{\mathrm{a}}$ & $53.25 \pm 6.15^{\mathrm{a}, \mathrm{b}}$ & $50.87 \pm 0.76^{\mathrm{a}, \mathrm{b}}$
\end{tabular}

Different letters represent statistically significant differences between any two data sets $(P<0.05)$.

(Bligh and Dyer, 1959; Stournas et al., 1995; Ryckebosch et al., 2012). Interestingly, unlike other investigations, which have found the Soxhlet extraction method to be a non-efficient method in recovery of many polyunsaturated fatty acids (Guckert et al., 1988), the current study shows promising results in both total FAMEs and total un-saturated fatty acids recovery when applied on pre-treated samples (Figure 5; Tables 3 and 4). In previous studies that used microwave pre-treatments for lipid extraction, an integrated microwave-Soxhlet extraction resulted in a high oil recovery rate, mainly due to the complete rupture of the cell structure by microwaves, which in turn caused a higher diffusion of algal oil into the extracting solvent (Choi et al., 2006; Balasubramanian et al., 2011). In the present study, thermal and microwave pre-treatments showed similar results in both the total lipid and total FAME recovery. It has been reported that the microwave pre-treatment has a lower rate of degradation of carotenoids present in olive oil and hence can produce a higher antioxidant-containing oil (El-Abassy et al., 2010; Balasubramanian et al., 2011). It should be noted that although the total lipid recovery of one extraction technique might show better results than others, the total amount of FAMEs derived from TAGs may be very different as shown by the results of this study. Considering the Soxhlet and Bligh \& Dyer extraction methods within the indirect transesterification experiments, the Soxhlet extractions showed considerably higher FAME recoveries for all of the pre-treatments.

An important consideration when improving the extraction efficiency is the intended use of the extracted lipids. A mix of saturated and the un-saturated fatty acids can produce a biodiesel with reasonably balanced fuel properties (Rashid et al., 2008). The saturated palmitic acid (C16:0) together with monounsaturated oleic acid (C18:1) have been suggested to be the most suitable indicators for the quality of biodiesel (Demirbas and Fatih Demirbas, 2011; Wiyarno et al., 2011). For both of these fatty acids, the Soxhlet extraction using a hexane:ethanol mixture on thermally pre-treated Tetraselmis sp. M8 biomass showed the best results. The promising fatty acid recoveries gained by the indirect transesterification by applying Soxhlet extraction on thermally pre-treated biomass should be considered along with reasonable extraction times ( $7 \mathrm{~h}$ including evaporation) in comparison with other reports on conventional Soxhlet applications that took 8-10 h (excluding evaporation time) (Virot et al., 2007; Balasubramanian et al., 2011). Direct transesterification has been reported to be more efficient than extraction-transesterification (indirect transesterification) (Johnson and Wen, 2009; Mercer and Armenta, 2011), but the present study shows a lower efficiency when compared with indirect transesterification techniques via Bligh \& Dyer or Soxhlet extraction when using pre-treated algal biomass (Figure 5). An added advantage is that none of the pre-treatment experiments in this study resulted in visible cell rupture and subsequent measurable release of the cell constituents including lipids into the media.

\section{CONCLUSION}

In conclusion, this study shows that thermally pre-treated, lyophilized algal biomass when extracted by Soxhlet using hexaneethanol (3:1), was the most efficient extraction method to recover fatty acids for biodiesel production from Tetraselmis sp. Direct transesterification was shown to be less efficient than indirect transesterification techniques when applied on pre-treated biomass. These conditions are amenable for scale-up and could be cost- and energy-efficient if solar-heating could be used for thermal pre-treatment followed by solar drying. Fine-tuning of the optimal parameters identified in this study may lead to further improvements of lipid and fatty acid extraction efficiencies.

\section{ACKNOWLEDGMENTS}

We wish to thank Dr. Matthew Timmins, Dr. Simon Tannock, Sourabh Garg, Lisa Bai, and Ali Malekizadeh for their technical support and useful discussions. We are grateful for financial support by the Australian Research Council and Meat \& Livestock Australia.

\section{REFERENCES}

Amin, S. (2009). Review on biofuel oil and gas production processes from microalgae. Energy Convers. Manag. 50, 1834-1840. doi:10.1016/j.enconman.2009.03. 001

Balasubramanian, S., Allen, J. D., Kanitkar, A., and Boldor, D. (2011). Oil extraction from Scenedesmus obliquus using a continuous microwave system-design, optimization, and quality characterization. Bioresour. Technol. 102, 3396-3403. doi:10.1016/j.biortech.2010.09.119

Bligh, E. G., and Dyer, W. J. (1959). A rapid method of total lipid extraction and purification. Can. J. Biochem. Physiol. 37, 911-917. doi:10.1139/059-099

Canakci, M., and Van Gerpen, J. (1999). Biodiesel production via acid catalysis. Trans. ASAE 42, 1203-1210. doi:10.13031/2013.13285

Chen, M., Liu, T., Chen, X., Chen, L., Zhang, W., Wang, J., et al. (2012). Subcritical co-solvents extraction of lipid from wet microalgae pastes of Nannochloropsis sp. Eur. J. Lipid Sci. Technol. 114, 205-212. doi:10.1002/ejlt.201100120

Chisti, Y. (2007). Biodiesel from microalgae. Biotechnol. Adv. 25, 294-306. doi:10. 1016/j.biotechadv.2007.02.001

Choi, I., Choi, S. J., Chun, J. K., and Moon, T. W. (2006). Extraction yield of soluble protein and microstructure of soybean affected by microwave heating. J. Food Process. Preserv. 30, 407-419. doi:10.1111/j.1745-4549.2006.00075.x

Cravotto, G., Boffa, L., Mantegna, S., Perego, P., Avogadro, M., and Cintas, P. (2008). Improved extraction of vegetable oils under high-intensity ultrasound and/or microwaves. Ultrason. Sonochem. 15, 898-902. doi:10.1016/j.ultsonch.2007.10. 009

Demirbas, A. (2008). Production of biodiesel from algae oils. Energy Sources 31, 163-168. doi:10.1080/15567030701521775

Demirbas, A., and Fatih Demirbas, M. (2011). Importance of algae oil as a source of biodiesel. Energy Convers. Manag. 52, 163-170. doi:10.1016/j.enconman.2010. 06.055

El-Abassy, R. M., Donfack, P., and Materny, A. (2010). Assessment of conventional and microwave heating induced degradation of carotenoids in olive oil by VIS Raman spectroscopy and classical methods. Food Res. Intern. 43, 694-700. doi:10.1016/j.foodres.2009.10.021

Gandhi, A., Joshi, K., JHA, K., Parihar, V., Srivastav, D., Raghunadh, P., et al. (2003). Studies on alternative solvents for the extraction of oil-I soybean. Int. J. Food Sci. Technol. 38, 369-375. doi:10.1046/j.1365-2621.2003.00683.x

Guckert, J. B., Cooksey, K. E., and Jackson, L. L. (1988). Lipid sovent systems are not equivalent for analysis of lipid classes in the microeukaryotic green alga, Chlorella. J. Microbiol. Methods 8, 139-149. doi:10.1016/0167-7012(88)90015-2 
Guillard, R. R. L., and Ryther, J. H. (1962). Studies of marine planktonic diatoms: I. Cyclotella nana Hustedt, and Detonula confervacea (Cleve) Gran. Can J. Microbiol. 8, 229-239.

Hejazi, M. A., and Wijffels, R. H. (2004). Milking of microalgae. Trends Biotechnol. 22, 189-194. doi:10.1016/j.tibtech.2004.02.009

Johnson, M. B., and Wen, Z. (2009). Production of biodiesel fuel from the microalga Schizochytrium limacinum by direct transesterification of algal biomass. Energy Fuels 23, 5179-5183. doi:10.1021/ef900704h

Kita, K., Okada, S., Sekino, H., Imou, K., Yokoyama, S., and Amano, T. (2010). Thermal pre-treatment of wet microalgae harvest for efficient hydrocarbon recovery. Appl. Energy 87, 2420-2423. doi:10.1016/j.apenergy.2009.11.036

Knothe, G. (2005). Dependence of biodiesel fuel properties on the structure of fatty acid alkyl esters. Fuel Process. Technol. 86, 1059-1070. doi:10.1016/j.fuproc.2004. 11.002

Lardon, L., Helias, A., Sialve, B., Steyer, J. P., and Bernardo, O. (2009). Life-cycle assessment of biodiesel production from microalgae. Environ. Sci. Technol. 43, 6475-6481. doi:10.1021/es900705j

Lee, J. Y., Yoo, C., Jun, S. Y., AHN, C. Y., and Oh, H. M. (2010). Comparison of several methods for effective lipid extraction from microalgae. Bioresour. Technol. 101, S75-S77. doi:10.1016/j.biortech.2009.03.058

Levine, R. B., Pinnarat, T., and Savage, P. E. (2010). Biodiesel production from wet algal biomass through in situ lipid hydrolysis and supercritical transesterification. Energy Fuels 24, 5235-5243. doi:10.1021/ef1008314

Lewis, T., Nichols, P. D., and McMeekin, T. A. (2000). Evaluation of extraction methods for recovery of fatty acids from lipid-producing microheterotrophs. J. Microbiol. Methods 43, 107-116. doi:10.1016/S0167-7012(00)00217-7

Lim, D. K., Garg, S., Timmins, M., Zhang, E. S., Thomas-Hall, S. R., Schuhmann, H., et al. (2012). Isolation and evaluation of oil-producing microalgae from subtropical coastal and brackish waters. PLoS ONE 7:e40751. doi:10.1371/journal. pone. 0040751

Luque-Garci'a, J., and Luque de Castro, M. (2004). Focused microwave-assisted Soxhlet extraction: devices and applications. Talanta 64, 571-577. doi:10.1016/ j.talanta.2004.03.054

Mercer, P., and Armenta, R. E. (2011). Developments in oil extraction from microalgae. Eur. J. Lipid Sci. Technol. 113, 539-547. doi:10.1016/j.copbio.2009.04.004

Moser, B. R. (2011). Biodiesel production, properties, and feedstocks. Biofuels 45(3):285-347. doi:10.1007/978-1-4419-7145-6_15

Patil, P. D., Gude, V. G., Mannarswamy, A., Deng, S., Cooke, P., Munson-McGee, S., et al. (2011). Optimization of direct conversion of wet algae to biodiesel under supercritical methanol conditions. Bioresour. Technol. 102, 118-122. doi:10.1016/j.biortech.2010.06.031

Pérez-Serradilla, J., Japon-Lujan, R., and Luque De Castro, M. (2007). Simultaneous microwave-assisted solid-liquid extraction of polar and nonpolar compounds from alperujo. Anal. Chim. Acta 602, 82-88. doi:10.1016/j.aca.2007.09.008

Rashid, U., Anwar, F., Moser, B. R., and Knothe, G. (2008). Moringa oleifera oil: a possible source of biodiesel. Bioresour. Technol. 99, 8175-8179. doi:10.1016/j. biortech.2008.03.066

Ryckebosch, E., Muylaert, K., and Foubert, I. (2012). Optimization of an analytical procedure for extraction of lipids from microalgae. J. Am. Oil Chem. Soc. 89, 189-198. doi:10.1007/s11746-011-1903-z
Sathish, A., and Sims, R. C. (2012). Biodiesel from mixed culture algae via a wet lipid extraction procedure. Bioresour. Technol. 118, 634-637. doi:10.1016/j.biortech. 2012.05.118

Stournas, S., Lois, E., and SerdariI, A. (1995). Effects of fatty acid derivatives on the ignition quality and cold flow of diesel fuel. J. Am. Oil Chem. Soc. 72, 433-437. doi:10.1007/BF02636084

Virot, M., Tomao, V., Colnagui, G., Visinoni, F., and Chemat, F. (2007). New microwave-integrated Soxhlet extraction: an advantageous tool for the extraction of lipids from food products. J. Chromatogr. A 1174, 138-144. doi:10.1016/ j.chroma.2007.09.067

Wahlen, B. D., Willis, R. M., and Seefeldt, L. C. (2011). Biodiesel production by simultaneous extraction and conversion of total lipids from microalgae, cyanobacteria, and wild mixed-cultures. Bioresour. Technol. 102, 2724-2730. doi:10.1016/j.biortech.2010.11.026

Wiyarno, B., Mohd Yunus, R., and Mel, M. (2011). Extraction of algae oil from Nannochloropsis sp.: a study of soxhlet and ultrasonic-assisted extractions. J. Appl. Sci. 11,3607-3612. doi:10.3923/jas.2011.3607.3612

Xu, L., Brilman, D. W. F., Withag, J. A. M., Brem, G., and Kersten, S. (2011). Assessment of a dry and a wet route for the production of biofuels from microalgae: energy balance analysis. Bioresour. Technol. 102, 5113-5122. doi:10.1016/j. biortech.2011.01.066

Yoo, G., Park, W. K., Kim, C. W., Choi, Y. E., and Yang, J. W. (2012). Direct lipid extraction from wet Chlamydomonas reinhardtii biomass using osmotic shock. Bioresour. Technol. 123, 717-722. doi:10.1016/j.biortech.2012.07.102

Zheng, H., Yin, J., Gao, Z., Huang, H., Ji, X., and Dou, C. (2011). Disruption of Chlorella vulgaris cells for the release of biodiesel-producing lipids: a comparison of grinding, ultrasonication, bead milling, enzymatic lysis, and microwaves. Appl. Biochem. Biotechnol. 164, 1215-1224. doi:10.1007/s12010011-9207-1

Conflict of Interest Statement: The authors declare that the research was conducted in the absence of any commercial or financial relationships that could be construed as a potential conflict of interest.

Received: 10 September 2014; paper pending published: 07 November 2014; accepted: 17 November 2014; published online: 04 December 2014.

Citation: Ghasemi Naghdi F, Thomas-Hall SR, Durairatnam R, Pratt S and Schenk $P M$ (2014) Comparative effects of biomass pre-treatments for direct and indirect transesterification to enhance microalgal lipid recovery. Front. Energy Res. 2:57. doi: 10.3389/fenrg.2014.00057

This article was submitted to Bioenergy and Biofuels, a section of the journal Frontiers in Energy Research.

Copyright (c) 2014 Ghasemi Naghdi, Thomas-Hall, Durairatnam, Pratt and Schenk. This is an open-access article distributed under the terms of the Creative Commons Attribution License (CC BY). The use, distribution or reproduction in other forums is permitted, provided the original author(s) or licensor are credited and that the original publication in this journal is cited, in accordance with accepted academic practice. No use, distribution or reproduction is permitted which does not comply with these terms. 